\title{
The relationship between perioperative nausea and vomiting and serum serotonin concentrations in patients undergoing cesarean section under epidural anesthesia
}

\author{
Byung Gun Lim ${ }^{1}$, Sang-Sik Choi ${ }^{1}$, Yu Jin Jeong ${ }^{1}$, Young Jin $\mathrm{Lim}^{2}$, Yong Chul Kim², Kyoung Un Park ${ }^{3}$, \\ Dong Kyu Lee ${ }^{1}$, and Mi Kyoung Lee ${ }^{1}$ \\ Department of Anesthesiology and Pain Medicine, ${ }^{1}$ Guro Hospital, Korea University College of Medicine, ${ }^{2}$ Seoul National University \\ College of Medicine, Seoul, ${ }^{3}$ Department of Laboratory Medicine, Seoul National University Bundang Hospital, Seoungnam, Korea
}

Background: Serotonin-also known as 5-hydroxytryptamine or 5-HT-can induce nausea and vomiting (NV) by peripheral mechanisms via the activation of 5- $\mathrm{HT}_{3}$ receptors. In this study, we observed perioperative NV, including intraoperative NV, and changes in serum 5-HT concentrations. We evaluated the relationship between perioperative NV and serum 5-HT levels in patients undergoing cesarean section under epidural anesthesia, and carried out a pilot study to determine if further studies on a larger scale were justified.

Methods: Twenty-eight patients who were scheduled for cesarean section under epidural anesthesia were included in the study. Patients were assigned to 2 groups according to the occurrence of NV after induction, i.e., an NV-positive or an NV-negative group. Serum 5-HT concentrations were measured before induction, at the time that NV occurred (in the case of the NV-positive group) or 5 min after the umbilical cord clamping (in the case of the NV-negative group) during surgery, and at $2 \mathrm{~h}$ postoperatively.

Results: NV occurred in 10 of the 28 patients. No significant differences in serum 5-HT concentrations were found within or between the two groups.

Conclusions: This study suggests that there is no correlation between serum 5-HT concentration and the occurrence of perioperative NV in patients undergoing cesarean section under epidural anesthesia, and the findings do not seem to support further investigations regarding a possible relationship between serum 5-HT concentration and perioperative NV. (Korean J Anesthesiol 2014; 67: 384-390)

Key Words: Cesarean section, Epidural anesthesia, Perioperative nausea and vomiting, Serum serotonin.

Received: April 1, 2014. Revised: 1st, June 5, 2014; 2nd, July 7, 2014. Accepted: July 11, 2014

Corresponding author: Sang-Sik Choi, M.D., Department of Anesthesiology and Pain Medicine, Korea University College of Medicine, Guro Hospital, 148, Gurodong-ro, Guro-gu, Seoul 152-703, Korea. Tel: 82-2-2626-1870, Fax: 82-2-851-9897, E-mail: clonidine@empal.com

(c) This is an open-access article distributed under the terms of the Creative Commons Attribution Non-Commercial License (http:// creativecommons.org/licenses/by-nc/3.0/), which permits unrestricted non-commercial use, distribution, and reproduction in any medium, provided the original work is properly cited. 


\section{Introduction}

Regional anesthesia is a highly effective, reliable, and safe anesthetic technique, and is currently the standard for elective and emergency cesarean section. However, despite its many advantages, a major drawback [1] is that perioperative nausea and vomiting (NV), a common and distressing side effect, develops in up to $80 \%$ of patients receiving regional anesthesia. It is well known that serotonin-also known as 5-hydroxytryptamine or 5-HT-plays a key role in the development of NV in many situations. Serotonin, which is markedly released from enterochromaffin (EC) cells in the gastrointestinal (GI) mucosa as a result of chemotherapy, may pass into the systemic circulation and act directly on central serotonin receptors in the vomiting center of the brain, causing chemotherapy-induced emesis (CIE) $[2,3]$. Serotonin receptor antagonists are very effective at preventing NV during regional anesthesia for cesarean section in parturients [4-6]. They act centrally by competitively and selectively binding to 5 -HT receptors in the chemoreceptor trigger zone of the central nervous system, as well as peripherally by blocking the receptors in the GI tract, thus preventing the action of 5-HT and inhibiting emesis [7]. Conversely, 5-HT can also act systemically and locally. If direct mechanical compression of the GI mucosa by a large gravid uterus and peripheral venous pooling induced by regional anesthesia cause substantial ischemia of GI mucosa, then a large amount of 5-HT may be released. The 5-HT that is released would pass into the systemic circulation, act directly on the central nervous system, and thereby cause NV by a mechanism similar to that of CIE. However, there are no reports that confirm a relationship between an increase in peripheral 5-HT and perioperative NV in patients undergoing cesarean section under regional anesthesia. In this study, we observed perioperative NV, including intraoperative $\mathrm{NV}$, and changes in serum 5-HT concentrations. We evaluated the relationship between perioperative NV and the serum 5-HT level in patients undergoing cesarean section under epidural anesthesia.

\section{Materials and Methods}

After obtaining the approval of our hospital's Institutional Review Board and written informed consent from the patients, 34 parturients, aged 22-37 years and of the American Society of Anesthesiologists (ASA) physical status II, who were undergoing elective cesarean section were enrolled in this study. They underwent epidural anesthesia without premedication for cesarean section. The patients who experienced NV during the intraoperative and immediate $2 \mathrm{~h}$ postoperative periods were categorized as the NV-positive group, whereas those who did not experience $\mathrm{NV}$ were categorized as the NV-negative group. The latter group consisted of patients who did not experience either nausea or vomiting since the first trimester of pregnancy, did not take any drugs known to interfere with the serotoninergic system within $48 \mathrm{~h}$ prior to the operation, and had avoided foods such as bananas and nuts, which are known to increase serotonin levels, from the day before the study. Patients were excluded if they had experienced severe gravid emesis, pre-eclampsia, or eclampsia. Patients with a psychological disorder or a cardiovascular, pulmonary, or endocrine disease were also excluded.

Standard electrocardiography and pulse oximetry monitoring were started once the patients arrived at the operating room. The radial artery was cannulated for blood samplings and blood pressure measurements under local anesthesia. The heart rate and blood pressure were recorded at $3 \mathrm{~min}$ intervals. All patients received a rapid infusion of $1,000-1,500 \mathrm{ml}(20 \mathrm{ml} / \mathrm{kg})$ of lactated Ringer's solution before an epidural injection of the local anesthetic. With the patient in the left lateral decubitus position, an $18 \mathrm{G}$ Tuohy needle was inserted into the L3-L4 interspace using a midline approach method. The epidural space was identified using the loss of resistance to air technique, and an epidural catheter was inserted $3 \mathrm{~cm}$ into the epidural space through a needle oriented in the cephalad direction. Since neither blood nor cerebrospinal fluid was obtained on aspiration, a $3 \mathrm{ml}$ test dose of $0.75 \%$ ropivacaine was injected. After 5 min without any signs of subarachnoid or intravenous injection, $17 \mathrm{ml}$ of $0.75 \%$ ropivacaine was injected. Aortocaval compression was avoided by maintaining patients in the supine position with a left lateral tilt. Oxygen was administered through a nasal cannula at a flow rate of $3 \mathrm{~L} / \mathrm{min}$.

Assessment for a sensory block and NV was performed by a study-blinded anesthesiologist. The completion of the epidural injection was taken as time 0 for all assessments. The sensory block level was checked by a pin-prick test every 5 min for up to 90 min after epidural injection. In all patients, the operation was started when a complete bilateral sensory block from T6 to S3 was achieved within $20 \mathrm{~min}$ of the epidural injection. If the sensory block from T6 to S3 was not achieved 20 min after the injection, $1.5 \mathrm{ml}$ increments of $0.75 \%$ ropivacaine per missing segment were injected epidurally, and the patient was excluded from the study.

Hypotension was defined as a systolic blood pressure below $100 \mathrm{mmHg}$ or as a systolic pressure reduction of more than $30 \%$ from the baseline. Hypotension was treated immediately with intravenous lactated Ringer's solution and ephedrine in $5 \mathrm{mg}$ increments. After delivery and clamping of the umbilical cord, 20 IU of oxytocin was added to $1,000 \mathrm{ml}$ of lactated Ringer's solution and allowed to infuse over a 45-60 min period.

The presence of NV was checked every 5 min during the operation. Nausea was defined as a subjective desire to vomit 
but without expulsive muscular movements. Vomiting was defined as an expulsive movement of the stomach muscles with or without expulsion of stomach contents. All episodes of postoperative NV experienced by the patients were also recorded until $2 \mathrm{~h}$ postoperatively in the postanesthesia care unit (PACU). Metoclopramide $10 \mathrm{mg}$ was injected intravenously as a rescue antiemetic. In order to prevent excessive sympathetic block, the epidural infusion pumps-with an infusion rate of $2 \mathrm{ml} / \mathrm{h}$ and a total volume of $100 \mathrm{ml}$ of $0.2 \%$ ropivacaine-were connected to the patients only when the sensory block decreased to the T8 level. Patients were not allowed to have clear liquids within the first $6 \mathrm{~h}$ or solid food within $24 \mathrm{~h}$ after surgery.

Blood samples were obtained before induction, at the time that NV occurred (in the case of the NV-positive group) or 5 min after the umbilical cord clamping (in the case of the NVnegative group) during the surgery, and at $2 \mathrm{~h}$ postoperatively. The arterial catheter was removed before the patient left the PACU, and the puncture site was compressed for $5 \mathrm{~min}$. Quantitative enzyme immunoassays for serum 5-HT concentrations were performed, according to the manufacturer's instructions, using Serotonin ELISA kits made by Immuno-Biological Laboratories in Hamburg, Germany.

Sample size calculation was based on the results of a pilot study performed with a total of 9 cases, 3 in the NV-positive group and 6 in the NV-negative group. One of the primary outcomes was that the serum 5-HT concentration of the second blood sample was $151.9 \pm 11.0 \mathrm{ng} / \mathrm{ml}$ in the NV-positive group and $140.8 \pm 3.9 \mathrm{ng} / \mathrm{ml}$ in the $\mathrm{NV}$-negative group, and also that the effect size of the 2 groups was 1.17 . On the assumption that the allocation ratio of $\mathrm{NV}$-negative group/NV-positive group was 2 , the total sample size was 28 ( 9 in the NV-positive group and 19 in the NV-negative group), the level of significance was 0.05 with a power of 0.8 as calculated by a two-sided Student's t-test, and the potential patient dropout rate was $20 \%$, the final sample size was increased to 34 .

Statistical analysis was performed using the Statistical Package for the Social Sciences (SPSS) data analysis software, version 15.0 for Windows (SPSS Inc., Chicago, IL, USA). The results are expressed as mean \pm standard deviation (SD) or median (range). Demographic data and anesthetic variables were analyzed using the Student's t-test or the Mann-Whitney U-test. Categorical variables were analyzed by using a chi-square test. To compare the serum 5-HT level within the NV-positive and negative groups, a one-way repeated measures analysis of variance was used, and post-hoc analyses were carried out using Scheffés method. To compare the serum 5-HT levels between the 2 groups at each sampling time and the rate of increment from the baseline, the Student's t-test or Mann-Whitney U-test was used to compare serum 5-HT concentrations to values obtained during the surgery between the groups. $\mathrm{P}<0.05$ was considered statistically significant.

\section{Results}

A total of 46 patients were originally eligible to participate in this study. Twelve patients were excluded for violations of the study protocol or for their refusal to participate. Thirty-four patients were subsequently enrolled in this study, but an additional 6 patients were further excluded for violations of the study protocol. Ultimately, 28 patients completed this study. The demographic and anesthetic data of the two groups were comparable in terms of age, body weight, height, body mass index, a history of perioperative NV or smoking, hypotensive events, ephedrine injection, and sensory block level (Table 1). There were no significant differences in the demographic and anesthetic variables between the groups. All patients were equally given 20 IU of

Table 1. Demographic and Anesthetic Data of Patients

\begin{tabular}{lcc}
\hline & NV - positive group $(\mathrm{n}=10)$ & NV - negative group $(\mathrm{n}=18)$ \\
\hline Age $(\mathrm{yr})$ & $31.0 \pm 3.0$ & $34.1 \pm 3.2$ \\
Weight $(\mathrm{kg})$ & $72.2 \pm 8.1$ & $67.6 \pm 11.3$ \\
Height $(\mathrm{cm})$ & $159.0 \pm 6.1$ & $158.0 \pm 3.8$ \\
Gestation (week) & $38.7 \pm 1.1$ & $38.3 \pm 0.7$ \\
Repeated cesarean section (n) & 5 & 10 \\
Multiparlity (n) & 5 & 10 \\
Smoking history (n) & 0 & 0 \\
Patients with hypotensive events (n) & 6 & 12 \\
Patients given ephedrine injection (n) & 5 & 0 \\
Previous history of perioperative NV (n) & 0 & $47.2 \pm 9.7$ \\
Duration of operation (min) & $51.3 \pm 14.5$ & $67.1 \pm 11.9$ \\
Anesthesia time (min) & $69.5 \pm 13.1$ & $\mathrm{~T} 4$ (T2-6) \\
Maximum segments blocked & $\mathrm{T} 3(\mathrm{~T} 1-6)$ & $24.4 \pm 10.0$ \\
Time to reach the highest block (min) & $25.8 \pm 11.1$ & 0 \\
\hline
\end{tabular}

Values are presented as mean \pm standard deviation (SD), median (range), or number of patients. NV-positive: patients with nausea or vomiting. NVnegative: patients without nausea or vomiting. NV: nausea or vomiting. T: thoracic level. 


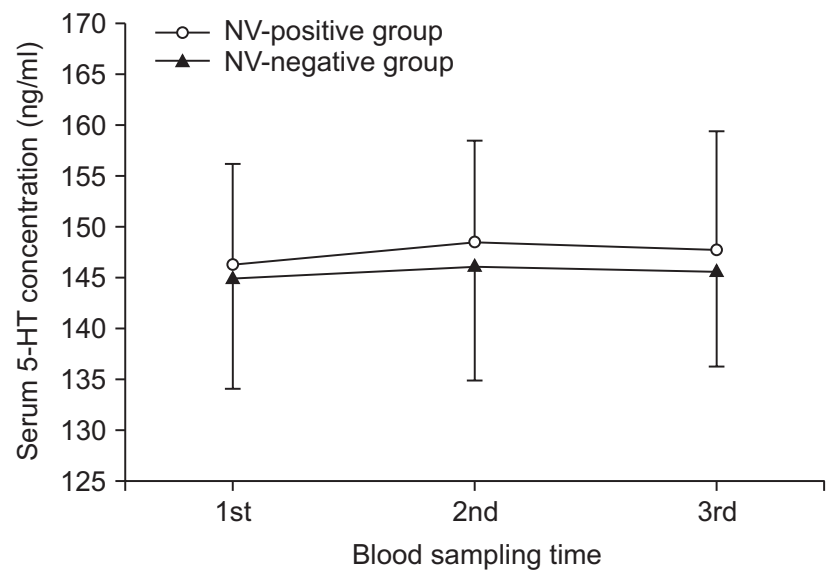

Fig. 1. The changes in serum 5-HT concentrations in the NV-positive and NV-negative groups. Values are presented as mean \pm standard deviation (SD). There were no significant differences between and within the two groups. NV-positive: patients with nausea or vomiting. NV-negative: patients without nausea or vomiting. 5-HT: 5-hydroxytryptamine (serotonin). 1st: before induction. 2nd: at the time of the occurrence of NV during the surgery (NV-positive group) or $5 \mathrm{~min}$ after the umbilical cord clamping (NV-negative group). 3rd: at $2 \mathrm{~h}$ postoperatively.

oxytocin as a uterotonic agent after delivery and clamping of the umbilical cord. No patient complained of significant visceral pain during the surgery. Ten out of the total of 28 patients (35.7\%) who experienced NV during the intraoperative and $2 \mathrm{~h}$ immediate postoperative periods were categorized as the NV-positive group, while the remaining 18 patients without $\mathrm{NV}$ comprised the NV-negative group. Among the 10 patients who experienced nausea during the study period, 6 experienced nausea during the intraoperative period, and 5 experienced nausea during the postoperative period. One patient experienced nausea during both intraoperative and immediate postoperative period. Four patients experienced vomiting: 2 during the intraoperative period and 2 during the postoperative period.

No significant changes could be seen in the serum 5 -HT concentrations within each group during the study period. In addition, there were no differences in the serial serum 5-HT levels between the 2 groups (Fig. 1). The mean serum 5-HT concentrations before induction, at the time of the occurrence of $\mathrm{NV}$ (in the case of the NV-positive group) or 5 minutes after the umbilical cord clamping (in the case of the NV-negative group) during the surgery, and at $2 \mathrm{~h}$ postoperatively were $146.3 \pm 9.8$ $\mathrm{ng} / \mathrm{ml}, 148.5 \pm 9.9 \mathrm{ng} / \mathrm{ml}$, and $147.8 \pm 11.6 \mathrm{ng} / \mathrm{ml}$ respectively in the NV-positive group and $144.8 \pm 10.8 \mathrm{ng} / \mathrm{ml}, 146.1 \pm 11.2 \mathrm{ng} /$ $\mathrm{ml}$, and $145.6 \pm 9.3 \mathrm{ng} / \mathrm{ml}$ in the $\mathrm{NV}$-negative group. The rate of increment between the first and second samples was also not different between the two groups: $1.7 \pm 5.2 \%$ in the NV-positive group and $1.1 \pm 6.6 \%$ in the NV-negative group.

\section{Discussion}

It is well known that the incidence of NV is high during both a cesarean section and a gynecological laparoscopic surgery $[1,8]$. However, the mechanisms are still unclear and are believed to be multifactorial $[9,10]$. In this study, there were no differences in the effects of the main causes (hypotension, uterotonic agent, surgical stimulation, and visceral pain) and the risk factors (age, sex, smoking, and a previous history of PONV) of PONV between the 2 groups. Therefore, in this study, it is highly possible that the action of 5-HT was associated with the occurrence of NV. Although many studies have shown that cytotoxic drugs evoke 5-HT release from the EC cells in the intestinal mucosa, and that the released 5-HT stimulates the 5-HT receptors on the adjacent vagal afferent nerves $[11,12]$, other studies have proposed that a large peripheral increase of 5-HT levels in CIE $[2,3]$ directly activates the area postrema of the brain. Such studies have demonstrated the beneficial effects of 5-HT antagonists in postoperative NV $[13,14]$; however, the relationship between perioperative NV and changes in serum 5-HT concentrations has not been elucidated.

Serotonin is a key modulator of gut function and when in excess, it can induce NV. The most important reservoirs of body serotonin are EC cells of the GI mucosa [15], which store more than $90 \%$ of the body's serotonin. Many physiological and pathological factors such as chemotherapeutic agents and luminal toxins such as cyclophosphamide, mechlorethamine, cisplatin, acids, alkalis, irritants, and bacterial toxins can induce NV because they result in the release of serotonin $[12,13]$. Mechanical GI mucosa stimulation, including distension, contraction, and surgical manipulation can also produce the same effect.

The increased serotonin release in CIE was demonstrated well by Cubeddu et al. $[11,16]$ using urine concentrations of 5-hydroxyindoleacetic acid (5-HIAA) and serum chromogranin A ( $\mathrm{CgA})$, metabolites of serotonin, as indices of serotonin release. Serotonin, which is released from EC cells in the GI mucosa by cytotoxic anticancer drugs, sends emetic signals to the vomiting center through the vagal afferent nerves in the GI mucosa. Serotonin may also pass into the systemic circulation and directly activate the area postrema, causing CIE [2,3]. Moreover, several established anti-emetic agents, such as ondansetron, granisetron, tropisetron, dolasetron, and palonosetron, are selective serotonin receptor antagonists $[2,12,17]$, which many studies have shown to be effective in the prevention of postoperative NV $[13,14]$. However, the relationship between 5-HT and perioperative NV remains to be confirmed.

Several studies conducted by Borgeat et al. [18], Nicole et al. [19], and Rusch et al. [20] have evaluated the relationship between 5-HT and postoperative NV. These authors showed that the distension of intestinal loops created by a pneumoperito- 
neum during laparoscopic surgery did not cause increased production of 5-HT by EC cells in GI mucosa. In those studies, they measured and compared excretions of the serotonin metabolite, 5-HIAA. Although the studies produced consistent results, they had several limitations. Firstly, the changes in the concentration of 5-HIAA in a 24-h urine sample could not reflect serotonin changes at the time of NV development or during ischemic conditions during the operation. Secondly, the authors could not detect intraoperative NV under general anesthesia despite an expected release of large amounts of serotonin. Thirdly, the pneumoperitoneum induced during laparoscopic surgery may have been insufficient to cause compression and thus resulted in ischemic changes in the GI mucosa.

This study was designed to overcome the limitations mentioned above. Our investigation postulated that patients who undergo cesarean section under regional anesthesia would be more susceptible to perioperative NV because of the effects of a large uterus and regional anesthesia. We thought that direct mechanical compression of the GI mucosa by a large gravid uterus and peripheral venous pooling induced by regional anesthesia could cause substantial ischemia of the GI mucosa, thereby increasing peripheral serotonin secretion and NV. We thought that we could minimize the effects of many other causes of NV by using the standardized epidural technique, selecting non-obese patients with an ASA physical status of I or II, and arranging for the same surgeon to perform the cesarean section. Moreover, we supposed that we could observe intraoperative NV and confirm the correlation between NV and serum 5-HT changes at the time of NV, by directly measuring serum 5 -HT concentrations through an arterial line. However, our expectations were unmet, as no correlation between serum 5-HT changes and perioperative NV was found.

Our study had two critical limitations. Because the half-life of 5-HT within the plasma is $2.5 \mathrm{~min}$ [21], the urinary excretion of 5-HIAA and the plasma levels of 5-HIAA and CgA have been used as markers for 5-HT release from the EC cells. They are regarded as valuable tools in the study of CIE $[12,22]$; however, there is controversy surrounding whether the circulating levels of plasma 5-HT increase after treatment with cytotoxic drugs [23-25]. Part of the discrepancies may be related to the methodology used for the collection and preparation of the platelet-free plasma [26]. That is, because 5-HT is avidly and effectively taken up by platelets, its rapid uptake into platelets may account for the failure to observe increases in serum levels. In fact, in our study, there is a possibility of 5-HT uptake into platelets during sample processing before centrifugation. Castejon et al. [27] reported that cisplatin-induced increases in blood 5-HIAA were not associated with increases in 5-HT blood dialysates through the application of intravenous microdialysis techniques, which allowed the continuous monitoring of plasma 5-HT and 5-HIAA and circumvented the problems related to sample handling. They insisted that the majority of the 5-HT released from the EC cells would be metabolized to 5-HIAA within the intestinal wall and/or during its passage through the liver or lung, leading to large increases in plasma and urinary 5-HIAA, which are not associated with increases in plasma 5-HT. These results support the view that circulating 5 -HT does not play a role in the emetic response associated with cisplatin treatment; therefore, it was likely that plasma 5-HT would not be associated with perioperative NV in our study. However, we noticed that an increased 5-HT metabolism and excretion resulted in a decreased serum 5-HT concentration and an increased urine 5-HIAA excretion, despite increased 5-HT production during pregnancy [28]. We suppose that the increase in the basal plasma and urine 5-HIAA levels masked their increase during the surgery. Besides, Houghton et al. [29] reported an increased platelet-depleted plasma 5-HT concentration following meal ingestion in symptomatic patients with diarrhea-predominant irritable bowel syndrome (IBS). Dunlop et al. [21] also reported that platelet-poor plasma samples were obtained in patients with postinfectious IBS and healthy volunteers, immediately and from 30 to $240 \mathrm{~min}$ after the test meal was applied to the patients, resulting in significant increments in plasma 5-HT levels in both groups until $180 \mathrm{~min}$ after the test meal. Bearcroft et al. [30] indicated that although only about $1 \%$ of the 5 -HT released can be measured as free 5-HT within the plasma, this measure is increased in patients with diarrhea-predominant IBS. Cubeddu et al. [24] reported that the content and concentration of 5-HT in platelets did not increase after treatment with cisplatin, despite large increases in the excretion of urine 5-HIAA. Taken together, we judged that we could confirm the correlation between NV and serum 5-HT changes if we shortened sample processing until centrifugation, after directly and promptly measuring serum 5-HT concentrations through an arterial line at the time of NV. However, our expectations were not realized, as there was no significant increase of serum 5-HT concentration at the time of perioperative NV. Therefore, further studies using plasma or urine 5-HIAA and serum CgA measurements will be needed to clarify the relationship between perioperative NV and serum 5-HT concentrations in patients undergoing cesarean section under epidural anesthesia.

Another limitation was that as a result of power analysis using the serum 5-HT concentration values of the second blood samples, one of the primary outcomes in this study-the power value-was very low. Therefore, our findings should be validated in an even greater number of patients. Nevertheless, the negative results of the present study may be useful in presenting the limitation of our method for serum 5-HT measurement and in recommending other methods such as plasma 5-HIAA or CgA measurement in such patients, given the high cost and time nec- 
essary for further studies and the difficulty in recruiting patients undergoing cesarean section under epidural anesthesia.

In conclusion, the present study suggests that no correlation exists between serum 5-HT concentration and the occurrence of perioperative NV in patients undergoing cesarean section under epidural anesthesia. In addition, it does not support further investigation into a potential relationship between serum 5-HT concentration and perioperative NV.

\section{References}

1. Balki M, Carvalho JC. Intraoperative nausea and vomiting during cesarean section under regional anesthesia. Int J Obstet Anesth 2005; 14: 230-41.

2. Andrews PL, Rapeport WG, Sanger GJ. Neuropharmacology of emesis induced by anti-cancer therapy. Trends Pharmacol Sci 1988; 9: 33441.

3. Higgins GA, Kilpatrick GJ, Bunce KT, Jones BJ, Tyers MB. 5-HT3 receptor antagonists injected into the area postrema inhibit cisplatininduced emesis in the ferret. Br J Pharmacol 1989; 97: 247-55.

4. Pan PH, Moore CH. Comparing the efficacy of prophylactic metoclopramide, ondansetron, and placebo in cesarean section patients given epidural anesthesia. J Clin Anesth 2001; 13: 430-5.

5. Pan $\mathrm{PH}$, Moore $\mathrm{CH}$. Intraoperative antiemetic efficacy of prophylactic ondansetron versus droperidol for cesarean section patients under epidural anesthesia. Anesth Analg 1996; 83: 982-6.

6. Yazigi A, Chalhoub V, Madi-Jebara S, Haddad F, Hayek G. Prophylactic ondansetron is effective in the treatment of nausea and vomiting but not on pruritus after cesarean delivery with intrathecal sufentanil-morphine. J Clin Anesth 2002; 14: 183-6.

7. Bunce KT, Tyers MB. The role of 5-HT in postoperative nausea and vomiting. Br J Anaesth 1992; 69 (7 Suppl 1): S60-2.

8. Tseng LH, Liou SC, Chang TC, Tsai SC, Soong YK, Wong SY. A randomized blinded study of the incidence of postoperative nausea and vomiting in women after major gynecologic laparoscopic surgery. J Minim Invasive Gynecol 2006; 13: 413-7.

9. Borgeat A, Ekatodramis G, Schenker CA. Postoperative nausea and vomiting in regional anesthesia: a review. Anesthesiology 2003; 98: 53047.

10. Gan TJ. Risk factors for postoperative nausea and vomiting. Anesth Analg 2006; 102: 1884-98.

11. Cubeddu LX. Serotonin mechanisms in chemotherapy-induced emesis in cancer patients. Oncology 1996; 53 (Suppl 1): 18-25.

12. Minami M, Endo T, Hirafuji M, Hamaue N, Liu Y, Hiroshige T, et al. Pharmacological aspects of anticancer drug-induced emesis with emphasis on serotonin release and vagal nerve activity. Pharmacol Ther 2003; 99: 149-65.

13. McKenzie R, Kovac A, O'Connor T, Duncalf D, Angel J, Gratz I, et al. Comparison of ondansetron versus placebo to prevent postoperative nausea and vomiting in women undergoing ambulatory gynecologic surgery. Anesthesiology 1993; 78: 21-8.

14. Eberhart LH, Morin AM, Hoerle S, Wulf H, Geldner G. Droperidol and dolasetron alone or in combination for prevention of postoperative nausea and vomiting after vitrectomy. Ophthalmology 2004; 111: 1569-75.

15. Read NW, Gwee KA. The importance of 5-hydroxytryptamine receptors in the gut. Pharmacol Ther 1994; 62: 159-73.

16. Cubeddu LX, O'Connor DT, Hoffmann I, Parmer RJ. Plasma chromogranin A marks emesis and serotonin release associated with dacarbazine and nitrogen mustard but not with cyclophosphamide-based chemotherapies. Br J Cancer 1995; 72: 1033-8.

17. Karim F, Roerig SC, Saphier D. Role of 5-hydroxytryptamine3 (5-HT3) antagonists in the prevention of emesis caused by anticancer therapy. Biochem Pharmacol 1996; 52: 685-92.

18. Borgeat A, Hasler P, Fahti M. Gynecologic laparoscopic surgery is not associated with an increase of serotonin metabolites excretion. Anesth Analg 1998; 87: 1104-8.

19. Nicole PC, Trepanier CA, Lessard MR. Nausea and vomiting after laparoscopic surgery are not associated with an increased peripheral release of serotonin. Can J Anaesth 2002; 49: 453-7.

20. Rüsch D, Strasser C, Celik I, Lengkong M, Wulf H, Scholz J. Vomiting after gynecologic laparoscopy and under general anesthesia is associated with changes in excretion of serotonin metabolites. Anaesthesist 2004; 53: 228-34.

21. Dunlop SP, Coleman NS, Blackshaw E, Perkins AC, Singh G, Marsden CA, et al. Abnormalities of 5-hydroxytryptamine metabolism in irritable bowel syndrome. Clin Gastroenterol Hepatol 2005; 3: 349-57.

22. Cubeddu LX, O’Connor DT, Parmer RJ. Plasma chromogranin A: a marker of serotonin release and of emesis associated with cisplatin chemotherapy. J Clin Oncol 1995; 13: 681-7.

23. Barnes NM, Ge J, Jones WG, Naylor RJ, Rudd JA. Cisplatin-induced emesis: preliminary results indicative of changes in plasma levels of 5-hydroxytriptamine. Br J Cancer 1990; 62: 862-4.

24. Cubeddu LX, Hoffmann IS, Fuenmayor NT, Malave JJ. Changes in serotonin metabolism in cancer patient: its relationship to nausea and vomiting induced by chemotherapeutic drugs. Br J Cancer 1992; 66: 198-203.

25. Fukui H, Yamamoto M, Ando T, Sasaki S, Sato S. Increase in serotonin levels in the dog ileum and blood by cisplatin as measured by microdialysis. Neuropharmacology 1993; 32: 959-68. 
26. Cubeddu LX, Alfieri AB, Hoffmann IS. Clinical evidence for the involvement of serotonin in acute cytotoxic-induced emesis. In: Serotonin and the scientific basis of antiemetic therapy. Edited by Reynolds DJ, Andrews PL, Davis CJ: Oxford, Oxford Clinical Communications. 1995, pp 142-9.

27. Castejon AM, Paez X, Hernandez L, Cubeddu LX. Use of intravenous microdialysis to monitor changes in serotonin release and metabolism induced by cisplatin in cancer patients: comparative effects of granisetron and ondansetron. J Pharmacol Exp Ther 1999; 291: 960-6.

28. du Bois A, Schäfer CA, Vach W, Prömpeler H, Bauknecht T, Breckwoldt M. Effect of pregnancy and labor on serotonin metabolism. Geburtshilfe Frauenheilkd 1995; 55: 500-4.

29. Houghton LA, Atkinson W, Whitaker RP, Whorwell PJ, Rimmer MJ. Increased platelet depleted plasma 5-hydroxytryptamine concentration following meal ingestion in symptomatic female subjects with diarrhoea predominant irritable bowel syndrome. Gut 2003; 52: 663-70.

30. Bearcroft CP, Perrett D, Farthing MJ. Postprandial plasma 5-hydroxytryptamine in diarrhoea predominant irritable bowel syndrome: a pilot study. Gut 1998; 42: 42-6. 